\title{
The UK physician workforce: one-third at increased risk of death from COVID-19
}

\author{
Authors: Ajay M Verma, ${ }^{\mathrm{A}}$ Andrew F Goddard, ${ }^{\mathrm{B}}$ Donal O’Donoghue, ${ }^{C *}$ Nina Newbery, ${ }^{\mathrm{D}}$ Christopher Phillips ${ }^{\mathrm{E}}$ and \\ Nigel TrudgillF
}

\section{Introduction}

$21 \%$ of NHS staff are from Black, Asian and minority ethnic (BAME) backgrounds yet account for a disproportionately high number of medical-staff deaths from COVID-19. Using data from the published OpenSAFELY Collaborative, we analysed consultant physicians to determine those at increased risk of COVID-19 related death.

\section{Methods}

Data from 13,500 consultant physicians collected by the Royal College of Physicians were analysed to determine those at an increased risk of death from COVID-19, assuming no comorbidities.

Results

The data reveal a picture in which a third of consultant physicians have a hazard ratio (HR) $>1$ for dying from COVID-19; one in five have $\mathrm{HR}>2$; one in $11, \mathrm{HR}>3$; and one in $40, \mathrm{HR}$ $>4$. Of concern are the risks to male physicians aged $\geq 60$ with $\mathrm{HR}>3.8$. Sub-specialties including cardiology, endocrine and diabetes, gastroenterology, haematology, neurology and rheumatology have a greater risk profile due to high proportion of men, physicians of older age, and proportion of BAME individuals.

Conclusion

A third of consultant physicians have an increased risk of a COVID-19-related death, and one in five have a higher relative risk ( $H R>2$ ). The risk is mainly driven by age, gender, and ethnicity, the risk is highest in male consultant physicians over 60 , especially from BAME backgrounds.

\footnotetext{
Authors: Aconsultant gastroenterologist and physician, Kettering General Hospital NHS Foundation Trust, Kettering, UK and chair of the New Consultants Committee, Royal College of Physicians, London,

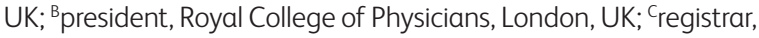
Royal College of Physicians, London, UK; Dhead of the Medical Workforce Unit, Royal College of Physicians, London, UK; E' Census design and production coordinator, Medical Workforce Unit, Royal College of Physicians, London, UK; F' Fonsultant gastroenterologist and physician, Sandwell and West Birmingham Hospitals NHS Trust, Birmingham, UK; * see note appending text
}

KEYWORDS: COVID-19, physicians, BAME, consultants, death

DOI: $10.7861 /$ clinmed.2020-0756

\section{Introduction}

Severe acute respiratory syndrome coronavirus 2 (SARS-CoV-2) was identified in 2019. Coronavirus disease 2019 (COVID-19) was characterised as a pandemic by the World Health Organization (WHO) on 11 March 2020 as it swept through Asia, Europe, the USA and Brazil, resulting in tens of thousands of deaths. ${ }^{1}$

The UK Office for National Statistics has reported that, after adjusting for age and other sociodemographic characteristics, and self-reported measures of health and disability, national data from March and early April 2020 revealed that COVID-19-related death for males and females of Black ethnicity was 1.9 times more likely than for those of White ethnicity. Similarly, males of the Bangladeshi and Pakistani ethnic groups were 1.8 times, and females 1.6 times, more likely to have a COVID-19-related death, while females in the Indian ethnic group were 1.4 times, and males 1.3 times, more likely to have a COVID-19-related death than those who are White. ${ }^{2}$

The OpenSAFELY Collaborative analysed 17 million primary care electronic health records within the UK NHS, including 10,926 deaths attributed to COVID-19. Cox-regression analysis was performed to generate hazard ratios (HRs) for COVID-19-related death adjusted for covariates. Death from COVID-19 was strongly associated with being male (HR 1.59), older age, deprivation and comorbidity, for example uncontrolled diabetes (HR 1.95). Compared to people of White ethnicity, Black (HR 1.48) and South Asian (HR 1.44) people were at higher risk of death. ${ }^{3}$

A more alarming trend has been noted among deaths of UK healthcare professionals from COVID-19. Black, Asian and minority ethnic (BAME) individuals account for approximately $21 \%$ of total NHS staff, including approximately $20 \%$ of nursing and support staff and $44 \%$ of medical staff. Shockingly, BAME individuals account for $63 \%$ of nursing, $64 \%$ of support staff and $95 \%$ of medical staff deaths. ${ }^{4}$

As a consequence of these data, it was recommended that individuals who may be at high risk of COVID-19 death should be risk-assessed, ${ }^{5}$ factoring in an individual's age, gender, ethnicity and comorbidities. The potential impact on the NHS and other 
Table 1. Numbers of consultant physicians by gender, ethnicity and age

\begin{tabular}{|c|c|c|c|c|c|c|c|c|c|}
\hline Female & $<34$ & $34-39$ & $40-44$ & $45-49$ & $50-54$ & $55-59$ & $60-64$ & $>65$ & \\
\hline White & 102 & 663 & 996 & 776 & 588 & 382 & 194 & 45 & $73.5 \%$ \\
\hline Mixed & 3 & 21 & 31 & 18 & 15 & 8 & 4 & - & $2.0 \%$ \\
\hline Asian & 22 & 217 & 347 & 253 & 148 & 47 & 21 & 6 & $20.8 \%$ \\
\hline Black & 1 & 16 & 18 & 22 & 15 & 12 & 3 & 1 & $1.7 \%$ \\
\hline \multirow[t]{3}{*}{ Other } & 1 & 13 & 33 & 32 & 16 & 5 & 2 & 1 & $2.0 \%$ \\
\hline & $2.5 \%$ & $18.2 \%$ & $28.0 \%$ & $21.6 \%$ & $15.3 \%$ & $8.9 \%$ & $4.4 \%$ & $1.0 \%$ & $37.8 \%$ \\
\hline & & & & & & & & & 5,098 \\
\hline Male & $<34$ & $34-39$ & $40-44$ & $45-49$ & $50-54$ & $55-59$ & $60-64$ & $>65$ & \\
\hline White & 67 & 526 & 895 & 854 & 954 & 838 & 635 & 246 & $59.7 \%$ \\
\hline Mixed & 3 & 26 & 30 & 34 & 24 & 14 & 11 & 4 & $1.7 \%$ \\
\hline Asian & 19 & 320 & 738 & 618 & 479 & 279 & 159 & 64 & $31.9 \%$ \\
\hline Black & 1 & 17 & 40 & 55 & 46 & 30 & 29 & 3 & $2.6 \%$ \\
\hline \multirow[t]{3}{*}{ Other } & 1 & 29 & 66 & 64 & 65 & 51 & 44 & 24 & $4.1 \%$ \\
\hline & $1.1 \%$ & $10.9 \%$ & $21.0 \%$ & $19.3 \%$ & $18.7 \%$ & $14.4 \%$ & $10.4 \%$ & $4.1 \%$ & $62.3 \%$ \\
\hline & & & & & & & & & 8,404 \\
\hline
\end{tabular}

healthcare systems of redeploying medical care healthcare staff who are at increased risk of death from COVID-19 from frontline work is unknown. We have therefore evaluated the risks of COVID-19-related death among 13,500 consultant physicians who work in the NHS.

\section{Methods}

The Medical Workforce Unit (MWU) of the Royal College of Physicians (RCP) collects census data annually of all consultant physicians working in the NHS in the UK. The last year of census data available is 2018 . Forms were sent electronically on 30 September 2018 to all substantive UK consultants. The MWU verified consultant numbers by working in partnership with the General Medical Council to check data for those UK consultants who are members or fellows of the RCP, RCPE (Edinburgh) or RCPSG (Glasgow). Additional consultant data were checked with national representatives of each medical subspecialty, and finally each hospital provider was telephoned to confirm data at a provider level. Data on total consultant numbers, specialty, workplace, gender and age are therefore as accurate as possible. Data on ethnicity are derived from census form responses and data held on members or fellows of the RCP, RCPE (Edinburgh) or RCPSG (Glasgow). ${ }^{6}$

\section{Analysis}

Using Microsoft Excel, risk grids based on age, gender and ethnicity were created. The hazard ratios (HR) applied to the risk grids were derived from the OpenSAFELY Collaborative's published data in the UK. ${ }^{3}$ Data on smoking, body mass index, deprivation and comorbidity were not available and it was therefore assumed for the purpose of the study that all individuals were non-smokers, nonobese, lived in the least deprived areas and had no comorbidities.

The data for all physicians within the NHS were analysed, including separate analyses of each major medical specialty. Consultant numbers were applied to the risk grid to evaluate the proportion of physicians with an increased risk of death from COVID-19 with $\mathrm{HR}>1, \mathrm{HR}>2, \mathrm{HR}>3$ and $\mathrm{HR}>4$.

\section{Results}

\section{Subjects}

Data on ethnicity and gender were available for 13,502 of 16,437 consultant physicians (82.1\%); two were excluded from the study due to incomplete age data. 5,099 (37.8\%) consultant physicians were female, of whom $26.5 \%$ were of BAME ethnicity. Of the $8,404(62.3 \%)$ male consultant physicians, $40.3 \%$ were of BAME ethnicity and $40.8 \%$ were aged 50 years or older (see Table 1).

\section{Risk grid for COVID-19-related death}

The risk grid constructed shows the calculated HR for UK individuals based on gender, age and ethnicity (see Fig 1). The reference is a White female aged $50-59$. Green represents $H R \leq 1$, amber $H R>1$ and $\leq 2$, and red with accentuation $H R>2, H R>3$ and $\mathrm{HR}>4$.

The sensitivity to age can clearly be seen, with a 2-6-fold increase in risk in those over 60 years of age. Men are at 1.59 times the risk of COVID-19-related death of women at any age. However, the effects of ethnicity are striking, with a doubling of the risk of COVID-19-related death in BAME men over 50 years of age and in BAME women over 60 years of age. There is a $5-6$-fold increased risk in BAME men over 60 years of age.

When calculating the risk for all consultant physicians combined using the data from Table 1 and Fig 1, 33.6\% have HR $>1$ of death from COVID-19, 18.4\% HR $>2,9.3 \% \mathrm{HR}>3$, and $2.5 \%$ $\mathrm{HR}>4$ (see Fig 2).

\section{Risk of COVID-19-related death among consultant} physicians from major medical subspecialties

Fig 3 includes data on the number of consultant physicians, their demographic breakdown and numbers at increased risk of COVID-19-related death in the largest medical sub-specialties in the UK. All sub-specialties have substantial numbers of consultants 


\begin{tabular}{|c|c|c|c|c|c|c|c|c|}
\hline Female & $<\mathbf{3 5}$ & $\mathbf{3 5 - 3 9}$ & $\mathbf{4 0 - 4 4}$ & $\mathbf{4 5 - 4 9}$ & $\mathbf{5 0 - 5 4}$ & $\mathbf{5 5 - 5 9}$ & $\mathbf{6 0 - 6 4}$ & $\mathbf{6 5}$ \\
\hline White & 0.06 & 0.06 & 0.30 & 0.30 & 1.00 & 1.00 & 2.40 & 2.40 \\
\hline Mixed & 0.09 & 0.09 & 0.43 & 0.43 & 1.43 & 1.43 & 3.43 & 3.43 \\
\hline Asian & 0.09 & 0.09 & 0.43 & 0.43 & 1.44 & 1.44 & 3.46 & 3.46 \\
\hline Black & 0.09 & 0.09 & 0.44 & 0.44 & 1.48 & 1.48 & 3.55 & 3.55 \\
\hline Other & 0.08 & 0.08 & 0.40 & 0.40 & 1.33 & 1.33 & 3.19 \\
\hline & & & & & & & \\
\hline Male & $<35$ & $\mathbf{3 5 - 3 9}$ & $\mathbf{4 0 - 4 4}$ & $\mathbf{4 5 - 4 9}$ & $\mathbf{5 0 - 5 4}$ & $\mathbf{5 5 - 5 9}$ & $\mathbf{6 0 - 6 4}$ & $>\mathbf{6 5}$ \\
\hline White & 0.10 & 0.10 & 0.48 & 0.48 & 1.59 & 1.59 & 3.82 & 3.82 \\
\hline Mixed & 0.14 & 0.14 & 0.68 & 0.68 & 2.27 & 2.27 & $\mathbf{5 . 4 6}$ & $\mathbf{5 . 4 6}$ \\
\hline Asian & 0.14 & 0.14 & 0.69 & 0.69 & 2.29 & 2.29 & $\mathbf{5 . 5 0}$ & $\mathbf{5 . 5 0}$ \\
\hline Black & 0.14 & 0.14 & 0.71 & 0.71 & 2.35 & 2.35 & $\mathbf{5 . 6 5}$ \\
\hline Other & 0.13 & 0.13 & 0.63 & 0.63 & 2.11 & 2.11 & $\mathbf{5 . 0 8}$ \\
\hline
\end{tabular}

Fig 1. Hazard ratios for COVID-19-related death in the UK population based on gender, ethnicity and age referenced to a 50 -year-old White woman.

at increased risk but this is particularly striking for cardiology, endocrinology and diabetes, gastroenterology and hepatology, haematology, neurology and rheumatology.

\section{Discussion}

The data reveal a concerning picture in which a third of UK consultant physicians have HR $>1$ of dying from COVID-19, one in five have $H R>2$, one in 11 have $H R>3$, and one in 40 have $H R$ $>4$. Of concern are the risks to all male physicians aged 60 or older, who all have $H R>3.8$. This risk is then exacerbated by factoring in ethnicity and personal comorbidities. Individualised risk profiles will show many at significant risk of death from COVID-19 compared to the benchmarked White woman aged 50-59 with no comorbidities. A similar pattern, albeit at lower order of magnitude, is seen in BAME male consultant physicians over 50 years of age, and BAME

\begin{tabular}{|c|c|c|}
\hline HR & \% & $\mathbf{n}$ \\
\hline $0-1$ & $66.4 \%$ & 8,958 \\
\hline$>1-2$ & $15.2 \%$ & 2,058 \\
\hline$>2-3$ & $9.1 \%$ & 1,227 \\
\hline$>3-4$ & $6.8 \%$ & 919 \\
\hline$>4$ & $2.5 \%$ & 338 \\
\hline & $100.0 \%$ & 13,500 \\
\hline
\end{tabular}

\begin{tabular}{|c|c|}
\hline $\mathrm{HR}>1$ & $33.6 \%$ \\
\hline $\mathrm{HR}>2$ & $18.4 \%$ \\
\hline $\mathrm{HR}>3$ & $9.3 \%$ \\
\hline $\mathrm{HR}>\mathbf{4}$ & $\mathbf{2 . 5 \%}$ \\
\hline
\end{tabular}

Fig 2. The stratification of risk to consultant physicians of a COVID-19-related death. female physicians over 60 years of age. These data strongly call into question whether these higher risk groups of older male physicians should be deployed in direct clinical care of patients with COVID-19 under any circumstances - even as vaccines become available (as they are not likely to be $100 \%$ effective).

Certain medical sub-specialties, including cardiology, endocrinology and diabetes, gastroenterology and hepatology, haematology, neurology and rheumatology are all notable due to the even higher risk profile of physicians in these specialties than consultant physicians in general. More men, older age and increased numbers of consultant physicians of BAME ethnicity contribute to the increased risk in these subspecialties.

In contrast, $<20 \%$ of consultants in acute medicine have $\mathrm{HR}>1$ due to a relatively younger consultant physician workforce in this subspecialty; $<25 \%$ of consultants in medical oncology and $<14 \%$ of palliative medicine consultant physicians have $\mathrm{HR}>1$ due to the high proportion of female consultant physicians in these specialties.

The hazard ratios formulated in this study are likely to be an underestimate of the risk, due to the assumption for the purpose of this study that all consultant physicians are non-obese, do not smoke and have no comorbidities. Although RCP London data show that doctors including consultant physicians are generally less obese, less likely to smoke, ${ }^{6}$ and have fewer comorbidities than the general population, individual consultant physicians need to adjust these risk estimates for additional personal risk factors. Furthermore, the hazard ratios do not include the unknown impact of increased exposure in specialities who are more likely to see patients with COVID-19, such as acute medicine and respiratory medicine, or for specialties which perform aerosolgenerating procedures, such as upper gastrointestinal endoscopy in gastroenterology.

It is important to remember that while male gender and BAME ethnicity confer higher risk of a COVID-19 related death, preliminary data from an RCP London survey do not show these individuals are more likely to contract COVID-19 compared to their female and White counterparts. 


\begin{tabular}{|c|c|c|c|c|c|c|c|c|}
\hline Specialty & $n$ & Age $\geq 50 \%$ & Female \% & BAME $\%$ & $H R>1 \%$ & $H R>2 \%$ & $\mathrm{HR}>3 \%$ & HR $>4 \%$ \\
\hline Acute medicine & 775 & 21.7 & 35.4 & 44.9 & 19.6 & 13.3 & 5.4 & 2.5 \\
\hline Cardiology & 1,349 & 43.7 & 14.3 & 37.1 & 40.7 & 19.6 & 9.6 & 2.5 \\
\hline Endocrine and diabetes & 930 & 42.9 & 36.0 & 47.8 & 36.6 & 20.0 & 9.2 & 1.5 \\
\hline Gastroenterology and hepatology & 1,312 & 42.6 & 21.5 & 35.7 & 37.8 & 17.7 & 7.9 & 1.7 \\
\hline Geriatric medicine & 1,389 & 41.2 & 43.9 & 37.0 & 34.4 & 22.2 & 11.2 & 4.9 \\
\hline Haematology & 743 & 45.6 & 49.7 & 29.7 & 35.9 & 20.3 & 9.8 & 2.6 \\
\hline Infectious diseases / tropical medicine & 207 & 42.5 & 38.2 & 15.0 & 34.8 & 15.5 & 11.6 & 2.9 \\
\hline Medical oncology & 461 & 32.5 & 49.8 & 24.0 & 23.9 & 9.1 & 4.3 & 0.2 \\
\hline Neurology & 754 & 46.8 & 30.0 & 27.3 & 39.1 & 18.3 & 11.1 & 1.7 \\
\hline Palliative medicine & 584 & 30.5 & 76.0 & 11.6 & 13.5 & 4.5 & 2.1 & 0.2 \\
\hline Renal medicine & 626 & 39.5 & 32.1 & 33.1 & 32.6 & 14.5 & 7.2 & 1.1 \\
\hline Respiratory & 1,252 & 36.2 & 30.8 & 33.7 & 31.4 & 16.9 & 8.5 & 2.0 \\
\hline Stroke medicine & 263 & 38.4 & 23.2 & 48.7 & 34.2 & 22.8 & 11.0 & 4.2 \\
\hline Dermatology & 695 & 42.3 & 60.7 & 35.1 & 29.6 & 16.8 & 8.1 & 1.0 \\
\hline Genitourinary medicine & 364 & 43.1 & 62.4 & 37.6 & 31.6 & 21.4 & 11.3 & 5.8 \\
\hline Rheumatology & 763 & 45.6 & 46.3 & 38.3 & 35.9 & 20.3 & 11.1 & 2.6 \\
\hline
\end{tabular}

Fig 3. Demographic details of each medical subspecialty within the UK and percentages with hazard ratios of COVID-19-related death.

Nevertheless, individual risk assessments need to be in place for all consultant physicians, especially those with increased exposure to COVID-19 patients and aerosol-generating procedures.

Factoring in age, gender and the presence of comorbidities such as class 3 obesity, poorly controlled diabetes, haematological malignancy or solid organ transplant (see Fig 4 ) is essential to make informed decisions on appropriate deployment of individual consultant physicians. This process needs to be uniform across the health service with a decision made at national level on what risk is deemed acceptable. The same risk assessment could then be used to decide which consultant physicians should be prioritised for
COVID-19 vaccinations, such as those at highest risk and working on the frontline, especially in higher-risk specialities and/or with exposure to aerosol-generating procedures. However, as vaccines are not $100 \%$ effective and may not be taken up by all, these risk profiles would remain relevant while COVID-19 is prevalent in the UK.

The risks from COVID-19 must be considered against a backdrop of a consultant physician workforce crisis in the UK, with $43 \%$ of consultant physician vacancies remaining unfilled, and $40 \%$ of consultant physicians stating that gaps in on call rotas occurred on a daily or weekly basis in their hospital.?

\begin{tabular}{|l|l|l|l|}
\hline Comorbidity & HR & Comorbidity & HR \\
\hline BMI & & Haematological malignancy & 2.82 \\
\hline Not obese (reference) & 1.00 & Diagnosed $<1$ year ago & 2.47 \\
\hline Obese class I $\left(30-34.9 \mathrm{~kg} / \mathrm{m}^{2}\right)$ & 1.05 & Diagnosed $1-4.9$ years ago & 1.62 \\
\hline Obese class II $\left(35-39.9 \mathrm{~kg} / \mathrm{m}^{2}\right)$ & 1.40 & Diagnosed $\geq 5$ years ago & 1.31 \\
\hline Obese class III $\left(\geq 40 \mathrm{~kg} / \mathrm{m}^{2}\right)$ & 1.92 & Diabetes & 1.95 \\
\hline Respiratory disease, excluding asthma & 1.78 & Controlled $(\mathrm{HbA} 1 \mathrm{c}<58 \mathrm{mmol} / \mathrm{mol})$ & 1.90 \\
\hline Stroke/dementia & 2.16 & Uncontrolled $(\mathrm{HbA} 1 \mathrm{c} \geq 58 \mathrm{mmol} / \mathrm{mol})$ & \\
\hline Other neurological disorder & 2.58 & No recent HbA1c measure & 1.72 \\
\hline Organ transplant & 3.55 & Reduced renal function & 2.52 \\
\hline Liver disease & 1.75 & CKD eGFR $30-60$ \\
\hline Chronic heart disease & 1.17 & CKD eGFR $<30$ \\
\hline
\end{tabular}

Fig 4. Selected comorbidities and associated hazard ratios for COVID-19-related death. ${ }^{3}$ 


\section{Conclusion}

Balancing the risk to the individual physician versus the needs of the patients within the understaffed NHS will be challenging, especially for the $18.4 \%$ who have a higher relative risk of dying from COVID-19. As the risk is mainly driven by age, gender, and ethnicity as the strongest risk factors (in that order), the risk is highest in male consultant physicians over 60 , especially those from a BAME background. Factoring in individual comorbidities such as class 3 obesity, poorly controlled diabetes, haematological malignancy and solid organ transplant also puts affected individuals at significant risk.

\section{Note}

During the course of submission and publication, Donal O'Donoghue, registrar for the Royal College of Physicians, contracted COVID-19 and sadly died from the infection on 3 January 2021, aged 64 . This paper is published in the spirit of Donal's intellectual endeavour, curiosity, and academic rigour. Rest in peace Donal.

\section{References}

1 World Health Organization. WHO Director-General's opening remarks at the media briefing on COVID-19 - 11 March 2020. WHO, 2020. www.who.int/dg/speeches/detail/who-directorgeneral-s-opening-remarks-at-the-media-briefing-on-covid-19-11march-2020.
2 Office for National Statistics. Coronavirus (COVID-19) related deaths by ethnic group, England and Wales: 2 March 2020 to 10 April 2020. ONS, 2020. www.ons.gov.uk/peoplepopulationandcom munity/birthsdeathsandmarriages/

3 Williamson EJ, Walker AJ, Bhaskaran $\mathrm{K}$ et al. Factors associated with COVID-19-related death using OpenSAFELY. Nature 2020;584: 430-6.

4 Cook T, Kursumovic E, Lennane S. Exclusive: deaths of NHS staff from COVID-19 analysed. Health Service J, 22 April 2020. www.hsj. co.uk/exclusive-deaths-of-nhs-staff-from-covid-19-analysed/ 7027471.article.

5 NHS Employers. Risk assessments for staff. NHS Employers, 2020. www.nhsemployers.org/covid19/health-safety-and-wellbeing/ risk-assessments-for-staff.

6 Royal College of Physicians. Hiding in plain sight: treating tobacco dependency in the NHS. RCP, 2018. Available from www.rcplondon. ac.uk/projects/outputs/hiding-plain-sight-treating-tobaccodependency-nhs.

7 Trudgill N. Focus on physicians. Census of consultant physicians and higher specialty trainees 2018. RCP, 2019. Available from www.rcplondon.ac.uk/projects/outputs/focus-physicians-2018-19census-uk-consultants-and-higher-specialty-trainees.

Address for correspondence: Dr Ajay M Verma, Department of Digestive Diseases, Kettering General Hospital NHS Foundation Trust, Rothwell Road, Kettering, Northamptonshire NN16 8UZ, UK.

Email:ajay.verma@nhs.net

Twitter: @UKGastroDr 